\title{
Intraoperative Computed Tomography Image Fusion for Orbital Blowout Fracture Reconstruction
}

Submitted: $6 / 7 / 2020$

Accepted: 28/10/2020 Online: 25/6/2021

\author{
Abd Jabar Nazimi*, Syed Nabil \\ Department of Oral and Maxillofacial Surgery, Faculty of Dentistry, \\ Universiti Kebangsaan Malaysia, 50300 Kuala Lumpur, Malaysia \\ *Corresponding author: mohdnazimi@ukm.edu.my
}

To cite this article: Nazimi AJ, Nabil S (2021). Intraoperative computed tomography image fusion for orbital blowout fracture reconstruction. Arch Orofac Sci, 16(1): 1-12. https://doi.org/10.21315/ $\operatorname{aos} 2021.16 .1 .1$

To link to this article: https://doi.org/10.21315/aos2021.16.1.1

\begin{abstract}
Intraoperative computed tomography (CT) has been previously described and acknowledged for its use in orbital blowout fracture reconstructions. We described a clinical case series managed by this technique combined with intraoperative image fusion for accuracy in orbital implant position. In total, eight patients who sustained a total number of 19 orbital wall fractures were described. From the total number of 19 blowout orbital fracture reconstructions comprised of medial and inferior (floor) orbital fractures, malposition was identified in a total of four orbital implants by using image fusion. All cases of implant malposition were immediately revised intraoperatively. Subsequent fusion was carried out to confirm whether the revision was satisfactorily achieved. We found that the intraoperative image fusion technique utilised to determine orbital implant position, especially at the posterior ledge, further augmented the role of intraoperative CT scanning. Image fusion conceptually provides an immediate, real-time, and objective solution for intraoperative image analysis and potentially eliminates problems with misaligned CT images. It also reduces the need for the surgeon to 'eye-ball' the CT images acquired or the need for additional intraoperative time, since the patient's head orientation is always axially at random during the acquisition of the CT. Conventional methods for CT image assessment are subjected to one's own interpretation and may introduce inconsistent or longer intraoperative decision-making. The technique facilitates intraoperative decision-making and reduces the risk of orbital implant malposition in orbital blowout fracture reconstructions. Hence, surgical complication in relation to orbital implant malposition in orbital blowout fracture management could be minimised. In addition, no further postoperative imaging is required.
\end{abstract}

Keywords: Image fusion; implant malposition; intraoperative imaging; orbital blowout fracture

\section{INTRODUCTION}

Orbital blowout fractures (OBFs) are common maxillofacial injuries (Ahmad Nasir et al., 2018) and can be challenging to manage (Manson et al., 1986). OBFs can be further divided into pure and impure types of fracture. Our previous study has shown that even a pure fracture, which is a small subset of OBFs, has significant prevalence at
13.8\% (Ahmad Nasir et al., 2018). Most of the fracture also involves critical intraorbital anatomical structures, such as the posterior ledge, transition zone and internal orbital fissure. In addition, the combination of these structures involved in $\mathrm{OBF}$ were also found to be substantial and lead to further complexity when it comes to their reconstruction due to the loss of anatomical landmarks. 
Complications following OBFs reconstruction surgery were also frequently seen (Schlittler et al., 2018). The overall complication rates reported widely vary from $3.0 \%$ up to $85.5 \%$, with $19.0 \%$ as the medium range (Gosau et al., 2011). Enophthalmos is a major complication of orbital fracture with key locations that lead to this was reported behind the eyeball equator (Zhang et al., 2012). To avoid surgical morbidity, OBFs reconstruction could benefit the most from reducing error and subjectivity when the surgeon utilises special considerations using the computer-assisted methods (Wan et al., 2015). Computerassisted techniques have been extensively described and have been proven beneficial not only for diagnostic purposes but also for its surgical management. The techniques include the use of pre-surgical computer planning (Jansen et al., 2018), intraoperative navigation (Shin et al., 2016; He et al., 2020), customised implant solutions (Lim et al., 2015) for true-to-original reconstructions (Schön et al., 2006) and intraoperative computed tomography (CT) scanning (Borad et al., 2017; Nazimi et al., 2019a). In addition, intraoperative CT has been described not only in maxillofacial surgical applications, such as in the management of zygomatic complex fracture (Cannizzaro et al., 2017; van Hout et al., 2014) and its concomitant orbital blowout fracture (Borad et al., 2017) but also in various others neurosurgical (Schichor et al., 2017) and orthopaedic surgical (Scarone et al., 2018) procedures.

It has been suggested that intraoperative CT plays an integral role in complex maxillofacial reconstruction. It allows real-time assessments and serves as valuable tool for intraoperative revisions, addressing suboptimal fracture reduction or malreduction, identifying displaced bony fragments (Stuck et al., 2012) or identifying malpositioning of implants (Borad et al., 2017). The revision can be done immediately within the same surgery, reducing the need for additional interventions and anaesthesia (Stuck et al., 2012).
In $\mathrm{OBFs}$ reconstruction, it was recently shown that intraoperative CT allows intraoperative evaluation of surgical repair, including orbital plate repositioning, orbital plate dimension and helping the surgeon to decide whether orbital exploration is required (Borad et al., 2017). Similarly, the study suggested that CT imaging served as a valuable tool in optimising orbital fracture repair and decreasing the dependency towards the need for postoperative CT scanning (Borad et al., 2017; Nazimi et al., 2019a).

However, the largest study looking at the use of intraoperative CT showed large variation ( $9 \%$ to $44 \%)$ regarding the need for intraoperative revision for both orbital and zygomatic complex (ZMC) fractures, as a wide range of assessments were simultaneously performed (van Hout et al., 2014; Borad et al., 2017). These findings must be viewed with caution, as they could be derived from non-standardised CT image analysis, analysis of non-aligned CT images, non-standard patient head orientation during the scanning procedure or manipulation of the CT scan image (which could be subjective based on self-interpretation or experience). In addition, the surgeon cannot simply provide continuous CT scanning following each revision without an increase in cumulative radiation exposure to their patient, although previous study has shown that an intraoperative CT scan imparts significantly less radiation than a standard CT scan (Zhang et al., 2009) and is sufficient to evaluate the surgical outcome with a low radiation dose (Cannizzaro et al., 2017).

The discrepancy in the scope of intraoperative decision-making needs to be addressed, since a previous study investigating preoperative CT scans showed that differences in diagnostic accuracy do exist between two- and threedimensional maxillofacial CT scan images and between expert and novice assessors (Jarrahy et al., 2011). Furthermore, the same could occur for the CT scan images derived intraoperatively. This cannot be 
overemphasised intraoperatively, as it requires a quick decision by the surgeon to benefit both the general anaesthetic and operating times. The aim of the present study was to elaborate our initial experience with the use of an intraoperative orbital implant image fusion of the CT images when intraoperative CT was utilised. The image fusion technique could provide further insight for more objective and accurate intraoperative image analysis for the final orbital implant position. Several fusion techniques were also described and highlighted of their potential benefits in facilitating intraoperative revision or decision-making.

\section{MATERIALS AND METHODS}

The present study comprised of a series of eight patients who sustained a total of 19 impure and pure OBFs that were treated in our facility from December 2018 to September 2019. OBFs involving the inferior (floor) and/or medial wall of the orbit were included in the study. Surgical consent was obtained, and all OBFs were reconstructed with pre-formed titanium orbital implant (Matrix Orbital; DePuy Synthes, Switzerland). The study was conducted in accordance with the Declaration of Helsinki adhering to medical protocol and ethics, and all patients consented for this routine surgical procedure. The present study was approved by the ethics committee of Universiti Kebangsaan Malaysia (approval number PPI/1 11/8/JEP-2020-639).

OBFs requiring surgical reconstruction were prepared in accordance with the computerassisted method, including pre-surgical virtual planning (Brainlab CMF iPlan 3.0.5, Brainlab ${ }^{\circledR}$, Feldkirchen, Germany), use of intraoperative navigation (Kick $₫$, Brainlab ${ }^{\circledR}$, Feldkirchen, Germany) and intraoperative imaging (O-arm Surgical Imaging System, Medtronic) in a low dose automated setting in the head region at $0.33 \mathrm{mSv}$.
Following orbital dissection, release of the herniated orbital contents and placement of the orbital implant under navigation, the first technique of intraoperative image fusion for the orbital implant was carried out between the pre-surgical planning and first intraoperative CT scan data to objectively determine the accuracy of fracture reconstruction. Four different locations were examined following the automatic image fusion of the orbital implant, namely the medial wall, transition zone, anterior aspect of the inferior wall (floor) and posterior aspect of the inferior wall (posterior ledge) utilising all axial, coronal and sagittal CT sections (Fig. 1). Orbital implant repositioning was carried out when indicated, and a second scan was performed at the surgeon's discretion in decision-making or when indicated, for example, when there was large or bodily implant adjustment. Following revision, both or either second or third technique of intraoperative fusion were carried out by using the data from three different scan types and used to finalise the treatment rendered to confirm the final orbital implant position.

\section{RESULTS}

In total, eight patients (all males) who sustained $19 \mathrm{OBFs}$ were included in the present study. There were nine pure and 10 impure $\mathrm{OBF}$ requiring $\mathrm{ZMC}$ fracture reduction prior to orbital reconstruction (Table 1). Of these eight patients, two patients sustained bilateral OBFs. All cases were secondary to trauma from motor vehicle accidents or assaults. For the fracture site, eleven left and eight right OBFs were treated. All cases were large OBFs involving both the inferior and medial wall without an intact transition zone, except in one case with an isolated pure inferior wall fracture. The main surgical indications for the surgical procedure were severe enophthalmos with globe motility restriction and diplopia towards the upper gaze. In all patients, the surgical approach was carried out via a pre-septal subciliary approach. 
With regard to the intraoperative imaging performed on these patients, four patients received a second intraoperative CT, as the position of the first attempt for the implant position within the orbit was deemed not satisfactory at the posterior ledge based on the first intraoperative CT. Orbital implant image fusion showed that the posterior ledge was the most common site for orbital implant malposition. In one patient, lateral implant malposition was detected, but the revision was made without performing a second intraoperative CT scan. In addition, all four cases with implant malposition at the posterior ledge were noted to have orbital fracture that involved the posterior ledge, making it difficult to determine the posterior location or end direction of the orbital implant under a limited direct or navigated procedure. In all, $50 \%(4 / 8)$ of the patients with $\mathrm{OBFs}$ required intraoperative orbital implant revision at the posterior ledge, or $26 \%(5 / 19)$ of all fractures when lateral malposition was included. We found no revision was required for the ZMC reductions in the impure fractures prior to placement of the orbital implant. In addition, there was no difference as to whether orbital plate revision was required in pure OBFs compared to impure OBFs.

From the perspective of reconstruction based on fracture sites, we found that no revision was required at three other critical intraorbital locations, namely the medial wall, transition zone and inferior wall of the orbit anteriorly. Adequate reconstruction at these critical orbital zones was satisfactorily achieved with the use of intraoperative navigation without the need for a second intraoperative CT or implant revision. The second and third image fusions carried out for the four cases requiring orbital implant

Table 1 Profiles of fracture locations and types

\begin{tabular}{lccc} 
Types of fracture & $\begin{array}{c}\text { Medial } \\
\text { wall }\end{array}$ & $\begin{array}{c}\text { Inferior } \\
\text { wall }\end{array}$ & Total \\
\hline Pure & 4 & 5 & 9 \\
Impure & 5 & 5 & 10 \\
\hline
\end{tabular}
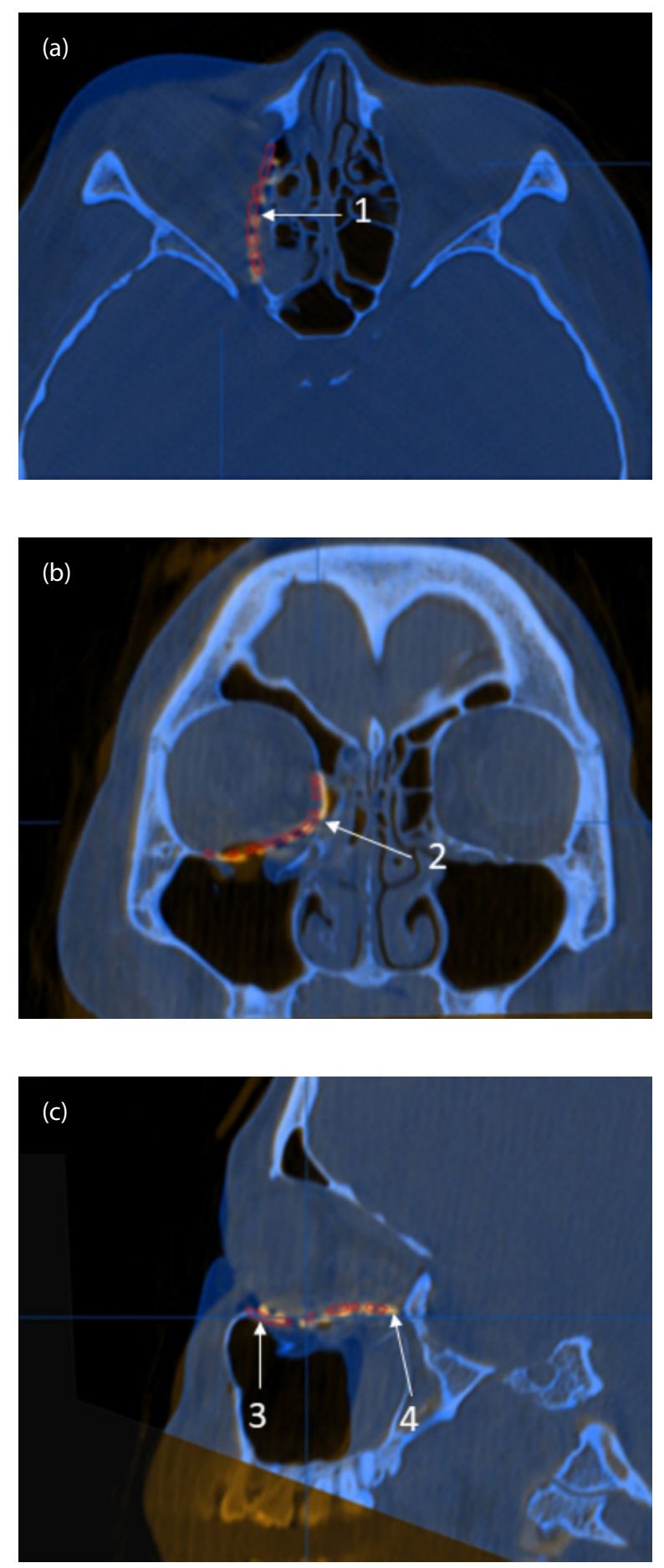

Fig. 1 Different locations to examine automatic image fusion of the orbital implant, utilising:

(a) axial; (b) coronal; (c) and sagittal CT sections. 
revision at the posterior ledge confirmed the findings of the first image fusion. None of our patients had an indication for postoperative imaging, although they presented with upper gaze restriction and diplopia during the early stage of postoperative recovery prior to the discharge date. No major enophthalmic complication was seen during the postoperative review duration of 4 to 32 weeks, except for disturbance in upward gaze limitation that was especially observed in delayed surgical repair. All patients were still under outpatient follow-up for a minimum duration of 48 weeks as per our institution review protocol.

\section{DISCUSSION}

OBFs may cause debilitating functional and aesthetic complications, such as diplopia and enophthalmos, due to entrapment of the extraocular tissues, muscles, or fat within the fracture site (Jin et al., 2000, Ahmad Nasir et al., 2018). OBFs can be divided into pure and impure types of fracture. In a pure $\mathrm{OBF}$, only the internal orbital wall (Lang, 1889; Smith \& Regan, 1957), such as the floor or inferior or medial wall of the orbit is involved (Hazani \& Yaremchuk, 2012), without involvement of the orbital rim or other regions. Our previous study showed that even a pure fracture, which is a small subset of OBFs, has significant prevalence at $13.8 \%$ (Ahmad Nasir et al., 2018).

The use of pre-formed titanium mesh with intraoperative control by using navigational surgery and postoperative CT imaging has been described in order to verify the exact three-dimensional reconstruction of the orbital cavity in achieving true to original fracture reconstruction (Schön et al., 2006) and reducing the risk of orbital implant malposition (Borad et al., 2017).

To date, application of computer-assisted surgery (CAS) techniques has been regarded and practiced as part of the surgical routine in posttraumatic orbital reconstructions
(Gellrich et al., 2002; Zizelmann et al., 2007). However, there is still limited availability for routine use of threedimensional intraoperative CT by many orbital trauma and maxillofacial surgeons. Currently, it may also not be readily available for many institutions, with costs and/or the amount of radiation exposure as primary concerns. In recent publications, intraoperative $\mathrm{CT}$ has been regarded as an essential tool for the facial reconstructive surgeon in optimising surgical repair (Borad et al., 2017; Nazimi et al., 2019a). The use of intraoperative CT with $\mathrm{O}$-arm also suggested that the imaging led to a significant change in operative management (Borad et al., 2017). Contrasting surgical practice or belief, it was also suggested that intraoperative CT may bring additional benefit in reducing radiation exposure and lessen the need for postoperative CT scanning that may inadvertently subject the patient to a higher radiation dosage in order to verify the final or postoperative position of the orbital implant and carries risk for the revisional procedure. Another study using an intraoperative cone beam CT (CBCT), which is a concept similar to the intraoperative $\mathrm{O}$-arm $\mathrm{CT}$, also suggested lower radiation exposure to patients but similarly led to a slightly higher percentage $(26 \%)$ of immediate intraoperative revisions (Stuck et al., 2012).

To the best of authors' knowledge, the state of current literature demonstrating or examining the intraoperative use of presurgical digital (in the form of imported stereolithography [STL]) and intraoperative CT scan data in OBFs reconstruction was never published in local medical or dental publications. Therefore, we further elaborate the intraoperative mirror technique in orbital trauma between the pre-surgical plan imaging data and intraoperative CT data that was first, but briefly, described earlier (Bittermann et al., 2014). In addition, the image fusion carried out in this early study briefly showed bony image fusion and orbital implant position in the coronal section but lacked further assessment at the posterior ledge of the orbit. 
We further highlighted the digital workflow comprising the second image fusion method by using intraoperative implant image data and its important intraoperative implications, especially when all coronal, sagittal and axial sections were concurrently analysed, aiming for high accuracy in the final orbital implant position. It is also imperative to understand that, although with the use of computerassisted elements (such as pre-surgical planning and intraoperative navigation without the use of imaging), patients with OBFs who underwent reconstruction with titanium mesh are still at risk for implant malposition (Borad et al., 2017). Therefore, it is understandable that correlation for the final orbital implant position can be made intraoperatively with regard to the presurgical or virtual planning position via the image fusion technique. This can be further augmented or confirmed with simultaneous use of intraoperative navigation. As mentioned, the navigation procedure itself could not entirely prevent plate malposition, as the final implant malposition could still occur as a result of various operative conditions or variation in orbital anatomy. Orbital anatomy was also described as unique to the individual, although the general anatomy of the orbital cavity and implant design has been extensively described (Bittermann et al., 2014). This article further explains that the effect of the orbital length exists in a stretching of the $\mathrm{S}$-shaped bulge of the orbit. The longer or deeper the orbital cavity is, the less steep the orbital bulge at the orbital floor and vice versa. This anatomical uniqueness requires in depth understanding and precise reconstruction to prevent complications but rather difficult to be genuinely appreciated during the surgery or always in confirmation with anatomical or pre-formed orbital implants.

Secondly, previous studies have also shown that even with the use of intraoperative CT imaging, large variations were seen with regard to the needs of intraoperative revision for both orbital and ZMC fractures (Borad et al., 2017; van Hout et al., 2014, Nazimi et al. 2019a). This discrepancy in the scope of surgeon or operational decision-indecision should be addressed, especially in the intraoperative context. A previous imaging study even addressing the 'less stressful' situation of preoperative scan images showed that differences in diagnostic accuracy do exist between two- and three-dimensional maxillofacial CT scans and between expert and novice assessors (Zhang et al., 2009). Therefore, the extended description of image fusion techniques for orbital implant data elaborated within this small case series could provide further insight for this clinical situation and heightened intraoperative decision-making.

The purpose of the present study was to describe the use of the intraoperative orbital implant image fusion technique in the surgical management of OBFs. Three image fusion techniques were analysed by using iPlan CMF ver 3.0.5 software (Brainlab $\AA$, Feldkirchen, Germany), which depends on the number of intraoperative CT scans performed. Suggested indications for both pure and impure OBFs are further elaborated in Table 2. However, the implementation of the technique, in the author's opinion, is at the discretion of the operating surgeon.

It carries out an additional but simple task and offers another possible way to optimise the preoperative and intraoperative scans Digital Imaging and Communications in Medicine (DICOM) data during surgery. The author prefers to combine the use of first and third fusion technique since it provides good reference to the pre-surgical planning simulation combined with intraoperative information (Fig. 2 and Fig. 3). The second fusion can be carried out between the first and second intraoperative CT scan data or images and further confirms the revision applied, albeit in the absence of the CT information from the pre-surgical planning simulation (Fig. 4). Various options for orbital implant image fusion also depends on the number of intraoperative scans performed. In addition, the scan images can also be utilised, even in the absence of 
Table 2 Suggested image fusion techniques, types of CT data sets used and its suggested indications

\begin{tabular}{llll}
$\begin{array}{l}\text { Intraoperative } \\
\text { image fusion } \\
\text { technique }\end{array}$ & \multicolumn{1}{c}{$\begin{array}{c}\text { CT Scan data set } \\
\text { utilised }\end{array}$} & \multicolumn{1}{c}{$\begin{array}{c}\text { Suggested indications } \\
\text { for pure OBF }\end{array}$} & $\begin{array}{c}\text { Suggested indications for } \\
\text { impure OBF }\end{array}$ \\
$\begin{array}{l}\text { First fusion } \\
\text { technique }\end{array}$ & $\begin{array}{l}\text { Preoperative and first } \\
\text { intraoperative data }\end{array}$ & $\begin{array}{l}\text { To compare the first attempt for } \\
\text { orbital implant position with the } \\
\text { virtual pre-operative planning } \\
\text { position in pure OBF }\end{array}$ & $\begin{array}{l}\text { To compare adequacy of first } \\
\text { attempt of fracture reduction in } \\
\text { an impure OBF to the virtual } \\
\text { pre-operative planning position }\end{array}$ \\
$\begin{array}{lll}\text { Second fusion } \\
\text { technique }\end{array}$ & $\begin{array}{l}\text { First and second } \\
\text { intraoperative data }\end{array}$ & $\begin{array}{l}\text { To compare second attempt for } \\
\text { orbital implant position following } \\
\text { intraoperative revision of } \\
\text { dimension or position to the } \\
\text { first attempt procedure }\end{array}$ & $\begin{array}{l}\text { To compare second attempt for } \\
\text { fracture reduction to the first } \\
\text { attempt reduction procedure }\end{array}$ \\
$\begin{array}{ll}\text { Third fusion } \\
\text { technique }\end{array}$ & $\begin{array}{l}\text { Preoperative and } \\
\text { second intraoperative } \\
\text { data }\end{array}$ & $\begin{array}{l}\text { To compare final orbital implant } \\
\text { position to the virtual pre- } \\
\text { operative planning position }\end{array}$ & $\begin{array}{l}\text { To compare final fracture } \\
\text { reduction position to the virtual } \\
\text { pre-operative planning position }\end{array}$ \\
\hline
\end{tabular}

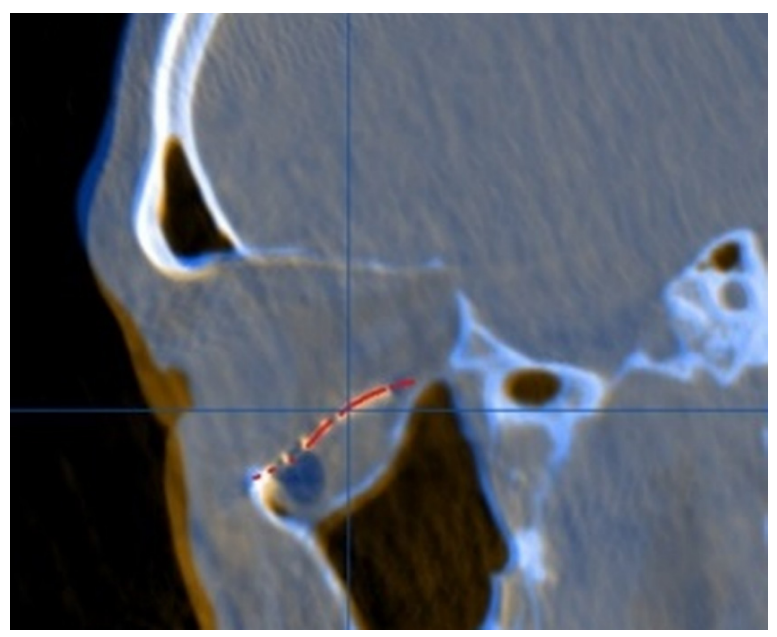

Fig. 2 Example of intraoperative image of first fusion between preoperative implant position data set (red outline) and intraoperative data set (orange) in sagittal view represent adequate implant position satisfactorily achieved during first attempt.

intraoperative navigation. However, it is likely that the amount of radiation exposure could increase when confirming orbital plate position, which can be checked much earlier to the pre-surgical plan if navigation-assisted procedures were used.

Additionally, another operative advantage with the use of intraoperative orbital implant image fusion techniques was reducing the high dependency towards the accuracy of the scanner isocentre position or dealing with non-standard head orientation of patients
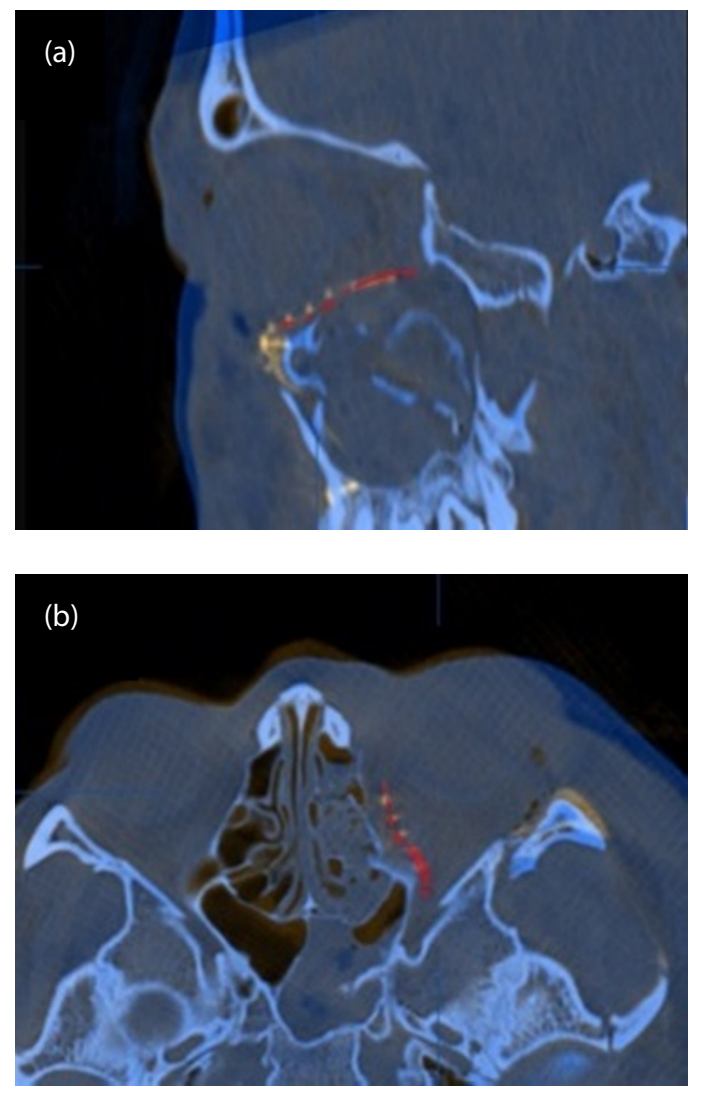

Fig. 3 Example of intraoperative image of third fusion between intraoperative data set implant position attempt 2 (yellow outline) and preoperative implant position data set (red outline), seen in sagittal (a) and axial (b) view represent adequate implant position following revision at the posterior ledge. Axial section of the scan also shows adequately reduced (yellow outline) left zygomatic complex fracture of an impure OBF. 


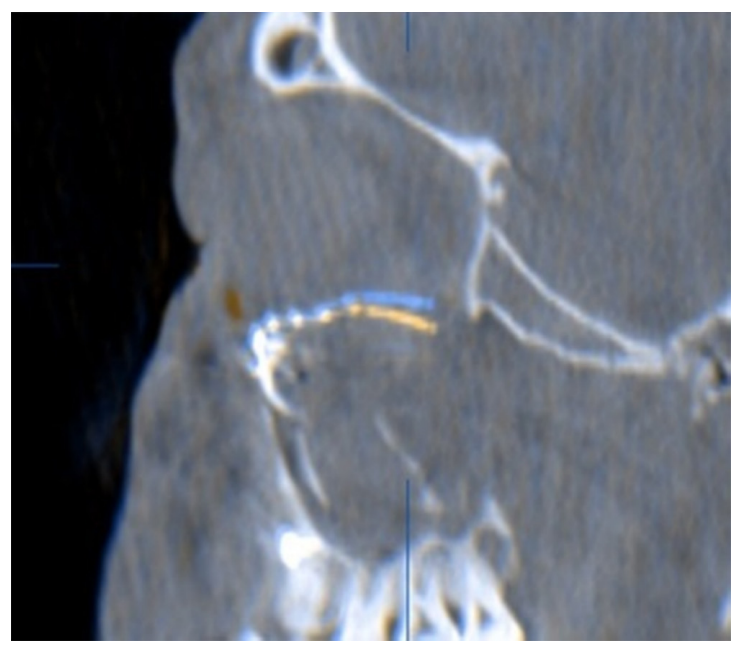

Fig. 4 Example of intraoperative image of second fusion between intraoperative dataset implant position attempt 1 (yellow outline) and intraoperative dataset implant position attempt 2 (blue outline) in sagittal view represent betterment in implant position at the posterior ledge following revision.

during the scanning procedure. With the use intraoperative CT scanning, moving the scanner to determine the isocentre is highly dependent upon training and may require significant operating time for adjustment, but it could never reach the same exact orientation.

However, in this small case series, the manoeuvring of the CT machine and scan duration were not recorded. With the image fusion technique, the image alignment can be performed automatically when the laser marking of the scan is placed within the surgical field of interest, which is the orbital bone. Manoeuvring of the CT machine and scan duration can be minimised, since accuracy is not warranted. If the surgeon him or herself was involved in the image fusion technique, the only intraoperative drawback was the requirement for the surgeon to temporarily break scrub to perform the task. This could be simply addressed by having trained personnel that could simultaneously perform the task, while the main surgeon remains in their surgical attire. Consequently, this could also save valuable operating time.
In the present study, we found that the CT was repeated in $50 \%$ of cases of OBF repair secondary to orbital implant malposition at the posterior ledge, which eventually required or led to intraoperative orbital implant revision. The percentage was slightly higher than $44 \%$ of cases that required a change in management, according to the recent large study using the same method (Borad et al., 2017). With the simultaneous use of intraoperative navigation, we found no requirement for implant revision at the medial wall, inferior wall and in transition zone of the orbit as confirmed by the intraoperative CT. We postulated that this could be secondary to the fact that the entire defect in these areas could be well visualised or examined with the use of direct view or navigation due to its 'favourable' anterior location and adequate surgical access. However, a similar conclusion could not be reached for the posterior ledge, although navigation was used. This is coupled with the fact that the posterior ledge could also be involved in the fracture and not be identified during orbital dissection. This was evidenced in CT images of cases requiring implant revision at the posterior ledge. This further emphasises our recent findings that described intraoperative navigation could only help the surgeon with intraoperative landmarks or positions but not the actual anatomical condition (Nazimi and Rajaran, 2019b). This may eventually lead to implant malposition, especially at the posterior ledge. It is not surprising to note that adequate exposure of the entire defect and visualisation of bony margins has been described and is important to guarantee accurate placement (Bittermann et al., 2014), but this surgical manoeuvre is rather difficult at the posterior ledge or when the posterior ledge or edge is also involved in the fracture. Aggressive orbital dissection may inadvertently contribute to postoperative surgical complications, such as orbital fat atrophy and injury to vital structures, and is best avoided whenever possible.

In addition, when comparing the use of intraoperative $\mathrm{O}$-arm $\mathrm{CT}$ and postoperative conventional CT or CBCT scan, we could 
appreciate that the radiological image qualities were found to be comparable in their quality addressing the surgical needs. The intraoperative CT imaging used in the present study was also based on the CBCT concept with automated low dose settings that are comparable to the use of intraoperative CBCT with lower radiation doses. Comparing both, it is interesting to note that both imaging techniques led to similarly high percentages $(26 \%)$ of detection of implant malposition in our series with immediate intraoperative revisions, as shown in a previous study (Stuck et al., 2012). The different intraoperative image fusion techniques elaborated potentially further enhance real-time and immediate intraoperative decision-making by surgeons that allow for the development of objective assessment and quality reconstruction with regards to patient diagnostic data and simulation. Without image fusion techniques, it is not known if the percentage is lower or higher secondary to subjective evaluations.

O-arm intraoperative $\mathrm{CT}$ as one of computer-assisted components in OBFs reconstruction has led to a positive change in operative management, even in our small series, as described above. This further emphasises the usefulness of this surgical tool in optimising orbital fracture repair and is in accordance with previous studies (Borad et al., 2017; Cannizzaro et al., 2017; van Hout et al., 2014, Nazimi et al., 2019a). From two-dimensional to three-dimensional images, the use of image fusion techniques could further enhance the potential of intraoperative CT imaging and has more to offer for primary delayed or for secondary OBFs reconstruction (Zizelmann et al., 2007; Heiland et al., 2004; 2005).

There were some limitations readily identified within the present study. First, the study comprised of only a small sample and was without long-term follow-up data. A total duration of 4 to 32 weeks postoperative follow-up in the management of OBFs, with its known injuries or surgical complications, is inadequate. Our institutional protocol will require a minimum follow-up period of 48 weeks, especially to detect any late enophthalmic complications. In addition, future study comprising not only the number of patients or cases treated but also comparing different fracture severity is suggested, as there is a dire need to ascertain what can be achieved intraoperatively with fracture reduction and plate position should provide good surgical outcomes for patients. It will also be beneficial for future study to look at the average time spent performing the image fusion procedure and the exact amount of radiation exposure for both patients and clinicians to weigh the risks and benefits of its usage. Oral and maxillofacial intraoperative $\mathrm{CT}$ and image fusion protocols could be established to reduce the radiation exposure of both patients and personnel, as shown in switching the dose technique for the paediatric spine $\mathrm{O}$-arm protocol (Su et al., 2016). Prospective, future study could also compare cases with intraoperative imaging and image fusion to cases that did not use intraoperative imaging and/or image fusion to determine the revision rate, surgical outcomes (such as plate position), surgical complications (such as diplopia and enophthalmos) and the rate of return visits to the operating room. On the other hand, the primary strength of the present study is the additional CT image fusion techniques described and its objective assessment value rather than subjectively 'eyeballing' the readily available digital CT data.

\section{CONCLUSION}

In summary, we have demonstrated several intraoperative image fusion techniques that are applicable with the use of pre-surgical virtual planning and intraoperative $\mathrm{O}$-arm CT dataset for more objective assessment of the final orbital implant position in OBFs reconstruction. The image fusion techniques described could further enhance the potential of computer-assisted methods for OBFs reconstruction and enhance intraoperative decision-making. It could reduce the 
subjectivity and/or variability in the image interpretation of surgeons. This prevents subjective intraoperative decision-making that is associated with human-related factors and potentially alleviates the complexity of the surgical procedure by introduction of a simple, yet useful, assessment method. The technique eliminates the need for the surgeon to 'eye-ball' the digital CT images that could be subject to one's own interpretation and inconsistency during both the scanning procedure and its assessment. Thus, the decision for intraoperative revision in orbital implant position or even orbital rim or outer frame reduction in impure OBFs reconstruction can be carried out more objectively. In addition, no further postoperative imaging was indicated, which directly influenced by the absence of postoperative uncertainties about the adequacy of reconstruction.

\section{ACKNOWLEDGEMENTS}

The authors would like to thank Medtronics (M) Sdn Bhd for the intraoperative CT scanner utilised in the management of OBFs cases illustrated in this manuscript, Brainlab (M) Sdn Bhd for image fusion via Iplan CMF Platform and Hospital Canselor Tuanku Mukhriz for the operating theatre.

\section{REFERENCES}

Ahmad NS, Ramli R, Nazimi AJ (2018). Predictors of enophthalmos among adult patients with pure orbital blowout fractures. PLoS One, 13(10): e0204946. https://doi.org/10.1371/journal. pone. 0204946

Bittermann G, Metzger MC, Schlager S, Lagrèze WA, Gross N, Cornelius CP et al. (2014). Orbital reconstruction: Prefabricated implants, data transfer, and revision surgery. Facial Plast Surg, 30(5): 554-560. https://doi.org/10.1055/s-0034-1395211
Borad V, Lacey M, Hamlar D, Dresner HS, Yadava GK, Schubert W (2017). Intraoperative imaging changes management in orbital fracture repair. f Oral Maxillofac Surg, 75(9): 1932-1940. https://doi.org/10.1016/j.joms.2017.05.002

Cannizzaro D, Revay M, Mancarella C, Colletti G, Costa F, Cardia A et al. (2017). Intraoperative imaging $\mathrm{O}-\mathrm{Arm}^{\mathrm{TM}}$ in secondary surgical correction of posttraumatic orbital fractures. Oral Maxillofac Surg Cases, 3(3): 56-60.

Gellrich NC, Schramm A, Hammer B, Rojas S, Cufi D, Lagrèze W et al. (2002). Computer-assisted secondary reconstruction of unilateral posttraumatic orbital deformity. Plast Reconstr Surg, 110(6): 1417-1429. https://doi.org/10 .1097/01.PRS.0000029807.35391.E5

Gosau M, Schoneich M, Draenert FG, Ettl T, Driemel O, Reichert TE (2011). Retrospective analysis of orbital floor fractures - Complications, outcome, and review of literature. Clin Oral Investig, 15(3): 305-313. https://doi.org/10.1007/ s00784-010-0385-y

Hazani R, Yaremchuk MJ (2012). Correction of posttraumatic enophthalmos. Arch Plast Surg, 39(1): 11-17. https://doi.org/10.5999/ aps.2012.39.1.11

He Y, Zhang Y, Yu GY, Guo CB, Shen GF, Peng X et al. (2020). Expert consensus on navigation-guided unilateral orbital fracture and orbital floor reconstruction techniques. Chin f Dent Res, 23(1): 51-55. https://doi. org/10.3290/j.cjdr.a44336

Heiland M, Schmelzle R, Hebecker A, Schulze $\mathrm{D}$ (2004). Intraoperative 3D imaging of the facial skeleton using the SIREMOBIL Iso-C3D. Dentomaxillofac Radiol, 33(2): 130-132. https://doi.org/10.1259/dmfr/ 15309653 
Heiland M, Schulze D, Blake F, Schmelzle $R$ (2005). Intraoperative imaging of zygomaticomaxillary complex fractures using a 3D C-arm system. Int $\mathcal{f}$ Oral Maxillofac Surg, 34(4): 369-375. https://doi .org/10.1016/j.ijom.2004.09.010

Jansen J, Schreurs R, Dubois L, Maal TJJ, Gooris PJJ, Becking AG (2018). The advantages of advanced computer-assisted diagnostics and three-dimensional preoperative planning on implant position in orbital reconstruction. f Craniomaxillofac Surg, 46(4): 715-721. https://doi.org/10.1016/j. jcms.2018.02.010

Jarrahy R, Vo V, Goenjian HA, Tabit CJ, Katchikian HV, Kumar A et al. (2011). Diagnostic accuracy of maxillofacial trauma two-dimensional and three-dimensional computed tomographic scans: Comparison of oral surgeons, head and neck surgeons, plastic surgeons, and neuroradiologists. Plast Reconstr Surg, 127(6): 2432-2440. https://doi.org/10.1097/PRS.0b013e31821 $3 \mathrm{a} 1 \mathrm{fe}$

Jin HR, Shin SO, Choo MJ, Choi YS (2000). Relationship between the extent of fracture and the degree of enophthalmos in isolated blowout fractures of the medial orbital wall. F Oral Maxillofac Surg, 58(6): 617-620. https://doi.org/10.1016/s02782391(00)90152-6

Lang W (1889). Traumatic enophthalmos with retention of perfect acuity of vision. Trans Ophthalmol Soc UK, 9: 41-45

Lim C, Campbell D, Cook N, Erasmus J (2015). A case series of rapid prototyping and intraoperative imaging in orbital reconstruction. Craniomaxillofac Trauma Reconstr, 8(2): 105-110. https://doi.org/10 $.1055 / \mathrm{s}-0034-1395384$

Manson PN, Clifford CM, Su CT, Iliff NT, Morgan R (1986). Mechanisms of global support and posttraumatic enophthalmos: I. The anatomy of the ligament sling and its relation to intramuscular cone orbital fat. Plast Reconstr Surg, 77(2): 193-202.
Nazimi AJ, Ching KS, Nabil S, Nordin R, Lan TH, Rajandram RK et al. (2019a). Intraoperative computed tomography scan for orbital fracture reconstruction f Craniofac Surg, 30(7): 2159-2162. https:// doi.org/10.1097/SCS.0000000000005667

Nazimi AJ, Rajaran JR (2019b). Re: Planes of reference for orbital fractures: A technique for reproducible measurements of the orbit on computed tomography scans. J Craniofac Surg, 30(3): 958. https://doi.org/ 10.1097/SCS.0000000000005297

Scarone P, Vincenzo G, Distefano D, Del Grande F, Cianfoni A, Presilla S et al. (2018). Use of the Airo mobile intraoperative CT system versus the O-arm for transpedicular screw fixation in the thoracic and lumbar spine: A retrospective cohort study of 263 patients. F Neurosurg Spine, 29(4): 397-406. https://doi.org/10 .3171/2018.1.SPINE17927

Schichor C, Terpolilli N, Thorsteinsdottir J, Tonn JC (2017). Intraoperative computed tomography in cranial neurosurgery. Neurosurg Clin N Am, 28(4): 595-602. https://doi.org/10.1016/j.nec.2017.05.010

Schlittler F, Schmidli A, Wagner F, Michel C, Mottini M, Lieger O (2018). What is the incidence of implant malpositioning and revision surgery after orbital repair? F Oral Maxillofac Surg, 76(1): 146-153. https://doi.org/10.1016/j.joms.2017.08.024

Schön R, Metzger M, Zizelmann C, Weyer $\mathrm{N}$, Schmelzeisen R (2006). Individually preformed titanium mesh implants for a true-to-original repair of orbital fractures. Int f Oral Maxillofac Surg, 35(11): 990995. https://doi.org/10.1016/j.ijom.2006.06 .018

Shin H, Kim S, Cha H, Han BL, Nam SM (2016). Real time navigation-assisted orbital wall reconstruction in blowout fractures. F Craniofac Surg, 27(2): 370-373. https://doi.org/10.1097/SCS.0000000000 002410 
Smith B, Regan WF Jr (1957). Blow-out fracture of the orbit: Mechanism and correction of internal orbital fracture. $\mathrm{Am}$ f Ophthalmol, 44(6): 733-739. https:/doi .org/10.1016/0002-9394(76)90774-1

Stuck B, Hülse R, Barth T (2012). Intraoperative cone beam computed tomography in the management of facial fractures. Int $\mathcal{F}$ Oral Maxillofac Surg, 41(10): 1171-1175. https://doi.org/10.1016/j.ijom.2012.06.006

Su W, Luo TD, McIntosh AL, Schueler BA, Winkler JA, Stans AA et al. (2016). Switching to a pediatric dose O-arm protocol in spine surgery significantly reduced patient radiation exposure. $\mathcal{F}$ Pediatr Orthop, 36(6): 621-626. https://doi. org/10.1097/BPO.0000000000000504

van Hout WM, Van Cann EM, Muradin MS, Frank MH, Koole R (2014). Intraoperative imaging for the repair of zygomaticomaxillary complex fractures: A comprehensive review of the literature. $\mathcal{F}$ Craniomaxillofac Surg, 42(8): 1918-1923. https://doi.org/10.1016/j.jcms.2014.07.012
Wan KH, Chong KK, Young AL (2015). The role of computer-assisted technology in post-traumatic orbital reconstruction: A PRISMA-driven systematic review. Sci Rep, 5: 17914. https://doi.org/10.1038/srep17914

Zhang J, Weir V, Fajardo L, Lin J, Hsiung H, Ritenour ER (2009). Dosimetric characterization of a cone-beam O-arm imaging system. F Xray Sci Technol, 17(4): 305-317. https://doi.org/10.3233/XST $-2009-0231$

Zhang Z, Zhang Y, He Y, An J, Zwahlen RA (2012). Correlation between volume of herniated orbital contents and the amount of enophthalmos in orbital floor and wall fractures. F OralMaxillofacial Surg, 70(1): 68-73. https://doi.org/10.1016/j.joms.2011 .02 .036

Zizelmann C, Gellrich NC, Metzger MC, Schoen R, Schmelzeisen R, Schramm A (2007). Computer-assisted reconstruction of orbital floor based on cone beam tomography. Br f Oral Maxillofac Surg, 45(1): 79-80. https://doi.org/10.1016/j.bjoms.2005.06.031 